\title{
Exilio interno y campo psi en Córdoba durante la última dictadura militar. Primeras aproximaciones e interrogantes
}

\section{Internal exile and psi scientific field in Córdoba in the last military dictatorship. First approaches and questions}

\section{Vissani, Laura; Scherman, Patricia; Fantini, Nilda ${ }^{1}$}

Resumen. Se busca comprender mejor la configuración del campo psi en Córdoba durante la última dictadura militar. Se analizan los espacios de encuentro y formación profesional que se desarrollaron y se discute si estos pueden ser definidos como prácticas de resistencia en condiciones de exilio interno. Se examina la noción de exilio interno en tanto enfatiza no sólo los desplazamientos de lugar geográfico, sino la mudanza de hábitos a la cuales los exilados se vieron sometidos. Se propone analizar la conformación de grupos de estudio -constituidos como estrategias alternativas de formación y lectura-, como prácticas de resistencia entendidas al modo foucaultiano. La figura de pliegue destacada por Deleuze, permite considerar la configuración de un espacio donde se hace posible eludir la violencia ejercida por el poder y continuar desarrollando un modo de hacer con otros, por fuera de los estrechos marcos regulatorios que imponía la dictadura.

Palabras Clave: Dictadura argentina - Psicología- Historia reciente- Exilio interno

\footnotetext{
${ }^{1}$ Vissani, Laura. Licenciada en Psicología, UNC. Profesora Asistente, Escuela de Ciencias de la Educación, UNC. Córdoba, Argentina. lauravissani@live.com.ar

Scherman, Patricia. Doctora en Psicología UNC. Profesora Titular Facultad de Filosofía y Humanidades, UCC, Facultad de Psicología UNC, Córdoba, Argentina. patoscherman@ gmail.com

Fantini, Nilda. Licenciada en Psicología, UNC. Maestranda, Maestría en Antropología,FFyH, UNC. Córdoba, Argentina. nildafantini@hotmail.com
}

Debates actuales en Psicología y Sociedad. ISSN en línea: 2591-524X

Año II, № 2, 2019. Pág. 1-16.

DOI: 10.22529/daps.2019.2(2)01 / Recibido: 19-05-2019 / Aprobado: 31-10-2019.

Artículo publicado bajo Licencia Creative Commons Atribución-NoComercial-SinDerivar. (c) Universidad Católica de Córdoba. 


\begin{abstract}
A better understanding of the psi scientific field configuration in Córdoba in the last military dictatorship is proposed. The meeting points and professional training are analyzed. We discuss if these can be defined as resistance practices in conditions of internal exile. This notion is examined, which emphasizes not only the displacements of geographical place, but the change of habits to which the people exiled were subjected. We propose to consider the formation of study groups -constituted at the time as alternative strategies for training and discipline-, as resistance practices understood in the foucaultian way. The fold figure highlighted by Deleuze, allows us to consider the configuration of a space where it is possible to avoid the violence exerted by power and continue to develop a way of working with others, outside of the narrow regulatory frameworks imposed by the military dictatorship.
\end{abstract}

Keywords: Argentine dictatorship - Psychology- Presente History- Internal exile 


\section{Introducción: Sobre el escenario que configuró la dictadura argentina en el campo psi}

El inicio de la dictadura militar que gobernó el país entre 1976 y 1983 significó un duro golpe para la ciudadanía, y también para la formación profesional en psicología en la Argentina. En tanto espacios de formación de jóvenes, las carreras de psicología fueron revisadas y evaluadas a la luz del nuevo enfoque que el gobierno de la dictadura buscaba implementar. Mientras algunas carreras cerraron sus puertas, ya sea de manera definitiva o por largo tiempo, en otras no se permitió el ingreso de nuevos estudiantes (Carpintero y Vainer, 2005; Calabresi, 2015; Di Doménico, Giuliani, Visca, Ostrovsky, Moya y Manso, 2008; Klappenbach, 2015; Sanz Ferramola, 2000). En Córdoba, tanto en la Universidad Nacional como en la Universidad Católica, se cerraron las puertas al ingreso de nuevos estudiantes, al mismo tiempo que se restringía el alcance de las lecturas permitidas (Autor, 2013; Autor, 2016). Algunos autores y textos fueron abiertamente censurados y otros, reducidos a una expresión puramente técnica, disminuidos en sus posibilidades de entrar en relación con otras lecturas, a riesgo de ser calificados de ideológicos, es decir, neutralizados en su capacidad creadora (Autor, 2018). "Las universidades se convirtieron en ámbitos acríticos donde se reprimió la libertad de pensamiento y fueron censurados o replanteados los programas de las carreras mientras se abandonaban las propuestas de formación y actualización profesional” (González Aguirre, 2012, p.117).

A la vez, se producía una masiva cesantía de docentes en las carreras ligadas a las humanidades y ciencias sociales. En Córdoba, las persecuciones a docentes y profesionales identificados con diversas propuestas transformadoras comenzaron en el año 1974, luego que el Gobierno Provincial fuera intervenido por el Gobierno Nacional, en un episodio conocido como el Navarrazo (San Nicolás, 2016; Servetto, 2004; Philp, 2009). Este golpe policial actuó con violencia directa sobre dirigentes estudiantiles y sindicales, dando como resultado muertes y numerosas detenciones. Esta violencia que el Estado ejerció de manera frontal hacia los sujetos involucrados en acciones de militancia política o social también impactó en las prácticas científicas y profesionales, en particular en los dispositivos de trabajo grupal y/o comunitario (Ferrero, Quiroga y Zuñiga, 2018). Tanto el trabajo de los colectivos profesionales como el interés hacia el cambio de las condiciones sociales en la labor comunitaria eran sospechados de dar lugar a actividades subversivas. En el ámbito de las prácticas ligadas al campo psi se disolvieron distintas iniciativas; se cerraron las incipientes 
comunidades terapéuticas, docentes y profesionales fueron separados de sus cargos o debieron partir al exilio (Carpintero y Vainer, 2005; Schiavoni y Wouters, 2014).

“...donde se hizo sentir de manera más demoledora el poder devastador de la fuerza represiva fue en los secuestros y desapariciones de profesionales y técnicos involucrados en proyectos y programas transformadores; en el recorte de espacios sociales y fundamentalmente en el doloroso desmantelamiento de las redes tejidas durante la puesta en práctica de estas experiencias, facilitadas por las novedosas orientaciones teóricas ingresadas por la psicología”. (González Aguirre, 2012, p.118)

Fue justamente en este período en que esta reciente profesión, que hacia fines de la década de 1960 encontraba que su rol comenzaba a afirmarse (Klappenbach, 2006), que comenzaba a ubicar su lugar y concebir su oficio en relación con la realidad latinoamericana (Autor, 2018), cuando la dictadura vino a interrumpir de manera violenta esos procesos. Klappenbach (2006) y Sanz Ferramola (2000) se refieren a los efectos de la dictadura sobre los movimientos y discusiones que se daban en ese momento en el campo psi, indicando que no sólo se secuestraron e hicieron desaparecer a psicólogos y estudiantes de psicología y se cerraron carreras universitarias de psicología, sino que a la vez se interrumpieron bruscamente las condiciones que habían favorecido el debate sobre el rol y que se visualizó a la psicología como una de las ideologías que habían promovido una visión ajena a la tradición del país.

Esta calificación de la psicología como "ideología”, que implica una descalificación de la misma como saber científico (Autor, 2016), alcanzó sobre todo a las prácticas que se apartaban del ejercicio individual de la profesión asociada de manera casi exclusiva al ámbito clínico, ejercicio que se percibía desvinculado de las acciones sociales y comunitarias, y ligado al tratamiento característico de la medicina. En aquel momento, los psicólogos que indagaban y se desempeñaban en otros ámbitos, que incursionaban en otras lecturas, que enriquecían e interrogaban los saberes recibidos, estaban comenzando a producir nuevas demarcaciones en el debate sobre el rol del este nuevo profesional, el cual comenzaba a concebirse como agente de cambio (Klappenbach, 2006).

En este marco, se producían relecturas de autores que habían sido recibidos en el país años antes bajo una impronta clínica, pero que a fines de la década de 1960 eran leídos y reflexionados desde otras coordenadas, sobre todo dentro de las universidades. Por ejemplo, 
las lecturas de Freud asociadas al marxismo, las lecturas de Piaget y de otros autores asociadas a las nuevas pedagogías, sobre todo a la pedagogía de la liberación, la teología de la liberación y la psicología social (Autor, 2018). Hacia principios de los años 1970 comenzaban a definirse nuevos sujetos y nuevas subjetividades, herederos del mayo francés de 1968 y de movimientos latinoamericanos, los cuales hacían sentir su efecto en las discusiones teóricas y en las prácticas profesionales, no desvinculadas de posicionamientos políticos (Autor, 2017). El texto Psicología, ideología y ciencia (Braunstein, Pasternac, Saal y Benedito, 1976) tan difundido en la UNC y en otros lugares de Latinoamérica, da testimonio de ello.

Esas trayectorias dispersas, pero presentes en distintos puntos del país, se interrumpen desde 1974 y más contundentemente a partir de 1976. El poder de la dictadura impone la necesidad de desistir en la continuación de ciertas orientaciones en las prácticas, particularmente aquellas que podrían llevar a un cuestionamiento del orden establecido. Al mismo tiempo, el gobierno militar suspendió la posibilidad de realizar reuniones, con lo cual quedaban también coartadas las posibilidades de conformar grupos profesionales, a riesgo de generar sospechas.

\section{El exilio y el insilio}

En este escenario, surge la dimensión del exilio, el cual hace referencia a la gran cantidad de personas que debieron optar por salir del país, buscando radicarse en algún país extranjero, a través de amigos u otros contactos (Jensen y Lastra, 2014). En el caso de nuestro estudio, algunos de estos profesionales retornarán luego al país y a las universidades a partir de 1984, reinsertándose a veces en los espacios de los que habían sido separados, y produciendo nuevas transformaciones, aun cuando los procesos interrumpidos en 1976 no podrán ser recuperados, en lo que se considera una inmensa pérdida.

Sin embargo, muchos profesionales que por diferentes razones no emigraron y se quedaron en el país, ya sea porque no se consideraban directamente perseguidos o porque no lograban salir del país para radicarse en el extranjero, bien porque no tenían los medios económicos o contactos materiales para hacerlo, sufrieron un impacto de otra índole. Nos referimos a aquellas personas que ante esta irrupción del régimen militar, se vieron obligadas por un lado a dejar su trabajo, ya sea por despido, autocuidado, o bien porque su trabajo se disolvió; que también soportaron el exilio o la desaparición de colegas, compañeros y seres queridos; que debieron modificar profundamente sus prácticas, y a veces mudarse de lugar de residencia, -barrio, ciudad o provincia-. "Frente al avasallamiento de las libertades 
individuales y la perspectiva de perder la vida, se desactivó todo liderazgo popular. El exilio, así como el insilio, fueron algunas de las estrategias más instaladas" (González Aguirre, 2012, p.118).

La denominación de exilio interno, que puede aparecer como contradictoria en su formulación, surge para dar cuenta de las migraciones o los desplazamientos que debieron realizar quienes vieron interrumpida una trayectoria de trabajo y de formación, sea porque se cerraron sus lugares de trabajo, o que fueron cesanteados, o que trabajaban con otros que habían partido al exilio (Casola, 2009). Algunos eligieron para desarrollar su profesión otros puntos del país, buscando nuevos espacios de trabajo, o bien tuvieron que mudar de prácticas laborales. Tal fue el caso de tres profesionales y docentes universitarias que entrevistamos en el curso de nuestra investigación, encontrándonos con esta particularidad que se repetía en las tres historias de vida: el desplazamiento, el movimiento, la búsqueda y creación de nuevos espacios donde desarrollar la profesión (Autor, 2015).

Se ha señalado que el exilio interno o insilio fue una modalidad más extendida entre la clase trabajadora y militantes de superficie (Casola, 2009). Sin embargo, aunque extendido, consideramos este que no ha recibido en nuestro campo la atención suficiente, dado que se discute la entidad del mismo y ciertas dificultades metodológicas lo acompañan. Entre las dificultades metodológicas ligadas a la investigación del exilio interno encontramos: ausencia de registros migratorios, inexistencia de colectivos de exiliados y de actividades específicas. De manera que el conocimiento de estos procesos descansa en la entrevista oral y las fuentes privadas como principal fuente de investigación (Casola, 2009).

Casola (2009) se pregunta por el modo en que el exilio interno podría, como el exilio exterior, recorrer el camino de la resistencia. Ya que, a diferencia de la comunidad de exiliados en el exterior que logró jugar un rol importante en las actividades de denuncia a la dictadura, creando redes de apoyo y solidaridad con los organismos de derechos humanos, desde la posibilidad de decir, de denunciar lo que estaban viviendo, los exilios internos estuvieron marcados por el silencio y la desconexión de las organizaciones. "Dado que la vida privada se convirtió en sustituto de la pública, el aislamiento o la autocensura se transformaron en opciones presentes en la vida cotidiana" (Gonzalez Aguirre, 2012, p. 118). El silencio y el ocultamiento como modalidad de lazo social que imponía la dictadura, a través de diversas estrategias, ha sido calificado como un proceso de inversión del mundo (Catela Da Silva, 2001). Esta autora -en su estudio sobre familiares de desaparecidos-, 
muestra cómo la tragedia individual, íntima, privada, transformó la relación con lo público. La práctica individual, privada, de la profesión, se convirtió en la alternativa más viable ante este escenario, lo cual se condice con lo planteado por Casola (2009) respecto de la preponderancia del ámbito privado en la definición de los nuevos modos de vida.

Sin embargo, si bien la opción de desvincularse y enfocarse en la práctica clínica en el ámbito privado parece haber sido la más recurrentemente adoptada en aquel momento en una Córdoba vigilada, para algunos profesionales fue posible producir algunas acciones que les permitieran poder continuar desarrollando, en parte, el camino y las lecturas elegidas. Nos referimos a la posibilidad de producir ciertos movimientos sobre una línea de acción, sobre el límite que la dictadura imponía, pero sin salirse de esa línea, ya que traspasarla implicaba la posibilidad concreta de persecución y muerte.

\section{Los grupos de estudio durante la dictadura}

Algunos de estos movimientos, que González Aguirre (2012) denomina como estrategias de supervivencia, fue la conformación de grupos de estudio. El progreso de la vida académica e intelectual ya no se centraba en las universidades, que se habían convertido en un territorio monótono en materia de desarrollo de pensamiento. Las carreras de psicología permanecían con una mínima actividad, despojadas de lecturas o discusiones transformadoras, escasamente innovadoras en materia de concepciones y prácticas del campo psi. Frente a este panorama surgían propuestas de lectura, grupales, pero pequeñas, de escaso número de participantes, los llamados grupos de estudio (Courel y Talak, 2001). Eran grupos más o menos cerrados, que se establecían en torno a un tema y/o autor en particular. En otros casos eran propuestas más abiertas, como cursos vinculados a asociaciones de profesionales, algunas recientemente creadas o actividades y espacios de formación convocadas desde los servicios de salud de los hospitales. Estos espacios se podían sustraer en parte a la mirada evaluadora de la censura, en tanto conservaran las características de perfeccionamiento técnico ligado siempre al ejercicio clínico de la actividad profesional.

González Aguirre (2012) se refiere a estas modalidades de agrupación de esta manera: "Fue en este contexto que muchos psicólogos "hicieron un refugio" en grupos de estudio, en función de diferentes problemáticas teóricas, pero, principalmente, amparados en la seguridad que proporcionaban vinculaciones con colegas cimentadas con anterioridad al golpe" (González Aguirre, 2012, p.122). Gentile (2009) ha destacado la importancia de la supervivencia de los vínculos, las micro-redes de reparación subjetiva y la defensa de los 
espacios sociales profesionales, tales como el hospital, los pequeños e itinerantes grupos de estudio, las asociaciones profesionales y las revistas. "A partir de estos grupos, y casi excluyentemente por afinidad teórica, se fortalecieron asociaciones científicas que contribuyeron, principalmente durante el período 1976-1978, a la unidad de la profesión" (González Aguirre, 2012, p.123). En Rosario, Gentile (2009) recuerda "el carácter vivificante del estudio y las discusiones que se dieron en ese periodo" en particular en las lecturas de Lacan, de su redefinición de la teoría y la clínica psicoanalítica. A estas experiencias asociativas se les reconoce un efecto positivo, ya que continuaron profundizando propuestas teóricas complejas, contribuyendo al desarrollo disciplinar local y fortaleciendo las bases científicas de la psicología (González Aguirre, 2012).

En esta misma línea, Diana Maffía (2010) reflexiona sobre su experiencia en sus años estudiantiles durante la dictadura y lo que para ella significó la Sociedad Argentina de Análisis Filosófico -SADAF-, fundada por profesores como Gregorio Klimovsky, que habían sido apartados de la universidad: "Las inolvidables reuniones de los sábados, donde una conferencia era seguida de un comentario y luego un debate colectivo, fueron una escuela de discusión crítica y aprendizaje de pluralidad y apertura." (Maffia, 2010, p.9). Nuestras entrevistadas han señalado algo semejante respecto a la intensidad del estudio, a la posibilidad de encontrar autores y enfoques novedosos, incluso acerca de la satisfacción alcanzada en el estudio reflexivo de los textos. Maffía ha señalado que en Sadaf cursó seminarios desde perspectivas teóricas, -materias, autores y métodos-, que no se transmitían en la carrera de filosofía de la UBA.En Buenos Aires, a estos grupos que mantuvieron vivo el conocimiento se los llamó después La Universidad de Las Catacumbas (Maffia, 2010, p.9).

Estas asociaciones y grupos de lectura, realizados al margen de las cátedras universitarias, surgieron como alternativa de formación en un momento y en un contexto particularmente opresivo, de censura en el terreno de las ideas y de persecución política. El momento particular en el que surgieron, esta coyuntura, nos lleva a considerarlos como espacios de resistencia, de creatividad, de potencialidad, que permitieron dar continuidad a las lecturas, a una libertad de pensamiento, y sostener las prácticas en el límite de lo permitido. Nos preguntamos ¿es legítimo pensarlas en tanto pliegues, en tanto formas de resistencia? Seguimos este interrogante, entendiendo a esta noción desde los desarrollos de Michel Foucault sobre el poder. 


\section{De poder y resistencias}

En el texto El sujeto y el poder, Foucault (1988) distingue entre relaciones de violencia y relaciones de poder:

Una relación de violencia actúa sobre un cuerpo o sobre cosas: fuerza, somete, quiebra, destruye: cierra la puerta a toda posibilidad. Su polo opuesto sólo puede ser la pasividad, y si tropieza con cualquier otra resistencia no tiene más opción que intentar minimizarla. En cambio, una relación de poder se articula sobre dos elementos, ambos indispensables para ser justamente una relación de poder: que "el otro" (aquel sobre el cual ésta se ejerce) sea totalmente reconocido y que se le mantenga hasta el final como un sujeto de acción y que se abra, frente a la relación de poder, todo un campo de respuestas, reacciones, efectos y posibles invenciones. (Foucault, 1988, p. 14)

En este sentido, consideramos que durante la dictadura iniciada en 1976 en la Argentina convivieron ambos modos: la violencia, que llevó a la desaparición forzada de personas, y un ejercicio del poder que restringió, censuró y prohibió ciertas prácticas individuales o grupales, pero sin anular totalmente las posibilidades de acción.

Es en este punto donde aparece la resistencia como una fuerza que surge en el seno de las relaciones de poder, pero que se despliega como una relación de provocación permanente; la resistencia como provocación, no como enfrentamiento del poder (Foucault, 1988, p.16). Es en este juego de poder y resistencia, de un ejercicio del poder que imponía estrechos márgenes al ejercicio de la libertad, donde no pudo aniquilar todas las diferencias, la resistencia encontró formas de manifestarse. Las ideas y las acciones que no eran permitidas encontraron la manera de sobrevivir bajo nuevas formas, de maneras diversas.

\section{Pliegues}

Podemos considerar a estas reuniones, asociaciones y grupos de estudio como una de esas formas de resistencia, justamente porque no se enfrentaron de manera directa al poder imperante, sino que se configuraron a la manera de un pliegue. Siguiendo a Deleuze en sus desarrollos sobre la subjetivación en Foucault, la noción de pliegue nos ayudará a despejar mejor, a precisar los rasgos de la aparición de estos espacios donde podía ejercerse cierta libertad, en un contexto de censura y restricción. Estos dispositivos, estas formas de resistencia surgidas en condiciones de exilio interno, pueden considerarse como un refugio, 
siempre que no lo entendamos como una vuelta hacia la interioridad de cada sujeto, a lo privado de cada uno, a una individualidad. En el afuera colectivo, en el ámbito de lo público, cualquier manifestación directa en oposición al poder, significaba arriesgar la vida. Proponemos considerar los grupos de estudio -de los cuales participaron estudiantes, académicos y profesionales- como pliegues. Siguiendo a Foucault, la subjetividad se distingue de una interioridad, para convertirse en un pliegue.

Se trata de las líneas primeras de una lectura posible ${ }^{2}$, que resulta interesante en tanto nos permite analizar estos dispositivos de formación sobre el trasfondo del momento y escenario en que surgieron y se desarrollaron, dejando huellas hasta hoy. Deleuze señala que al final de su período de reflexión sobre el poder, Foucault se pregunta por la posibilidad de franqueamiento de la línea del poder y cómo sería posible hacerlo, en los siguientes términos: “cómo alcanzar un afuera que sea verdaderamente un afuera?” (Deleuze, 2015, p. 14). La pregunta puesta en juego por Foucault es si se puede plantear un más allá de la línea del poder, si es posible traspasar el límite trazado por el poder, y advierte que más allá de ese límite lo que se presenta es el se muere, la muerte efectiva, pero también la muerte en el sentido de la no vida, de aquello que anula las posibilidades vitales. "En otros términos, habría efectivamente un más allá de la línea del poder, habría efectivamente franqueamiento del poder, habría efectivamente línea del afuera, pero este más allá aparece como la muerte" (Deleuze, 2015, p. 14).

No obstante, existe la posibilidad de que se produzca otra cosa en esa misma línea del afuera: “...para que todo sea relanzado sería preciso -aquí tampoco tenemos elección- que la línea del afuera sea capaz de ciertos movimientos que la arranquen de la muerte" (Deleuze, 2015, p. 21). Esta otra cosa a la que se alude es un movimiento, el cual puede sustraer una parte de esa línea del afuera, y producir una invaginación sobre esa línea, dando lugar a un pliegue. Esta línea del afuera es fundamentalmente movimiento, entendido como flujo, oscilación o vibración permanente. Metafóricamente se la ilustra bien como una línea animada por movimientos peristálticos, o bien como una invaginación, según el uso que a este término se le da en embriología. Una invaginación que va a formar un hueco, conformado por

\footnotetext{
${ }^{2}$ Nos apoyamos en el texto La subjetivación: curso sobre Foucault III, de Gilles Deleuze (2015), donde se reúnen las clases del curso que el filósofo dictó sobre Foucault en la Universidad de Vincennes, entre abril y mayo de 1986. Es el tercero y último de una serie de cursos sobre lo que él llama los tres ejes del pensamiento de Foucault: el saber, el poder, la subjetivación.
} 
el movimiento de la línea. Es en esa posibilidad de hacer un pliegue donde sería posible vivir en relación al horizonte del poder, el hueco donde algo de la vida pueda ser posible.

Es esta línea del afuera que al plegarse constituye un adentro más profundo que no remite a un mundo interior, genera un espacio a través de esta operación que Foucault llamará subjetivación.

De este modo, la subjetivación aparece como plegamiento y construcción de un espacio nuevo, diferente, que produce y aloja subjetividad, pero que se deriva de la línea del poder, de un afuera que es su límite. Aún dentro de un sistema atravesado por líneas de fuerza que traza el poder, a través de la censura, las prohibiciones, hay un espacio posible donde ejercer esa libertad, donde el gobierno de sí sea posible.

Aquí vemos entonces, desplegarse el tercer eje del pensamiento de Foucault: la subjetividad. Deleuze remarcará que el ser del sujeto, o la subjetividad, es el plegamiento del afuera (p.53); indica que, al plegarla, se arranca a la línea del afuera de la muerte y encuentra una manera de vivir, que es vivir en los pliegues. El poder en sus más variadas formas, se impone, forma parte de nuestras vidas, pero a su vez, la vida produce nuevos pliegues, nuevas formas de subjetivación que se sustraen a esas nuevas formas de poder, que tendrían potencial para generar espacios sustraídos de las amenazas por el control y la dependencia del poder. A su vez, una vez surgidas nuevas formas de subjetivación, el poder va a tratar de controlarlas, de doblegarlas.

La dimensión de la subjetivación es tanto individual como colectiva, dirá Deleuze (2015, p.171), y daba como ejemplos las subjetivaciones comunitarias de mayo del '68 así como la subjetivación de las mujeres con los movimientos de liberación femenina. La emergencia de nuevas luchas, de nuevos tipos de lucha y la emergencia de nuevas subjetivaciones son complementarias. La subjetividad no aparece como una interioridad individual, fuera de las coordenadas impuestas por el afuera, por lo social, por las relaciones de poder en un momento y contexto determinado. Al contrario, es atravesada por estas luchas ${ }^{3}$.

\footnotetext{
${ }^{3}$ Entre los tres ejes, poder, saber y sí mismo o subjetivación, habrá todo el tiempo relaciones de lucha, de oposición y también de compromiso. "Pero así como no cesan de nacer nuevas relaciones de poder, y así como no cesan de nacer nuevas formas de saber, tampoco cesan de nacer nuevos modos de subjetivación, capaces algunas veces de oponerse a las relaciones de poder y de independizarse de ellas, otras veces de entablar con ellas compromisos." (Deleuze, 2015, p.134).
} 
En conversación con Félix Guattari, en las mencionadas clases de 1986, este pensador, activista del mayo francés del '68 y coautor de varias obras junto con Deleuze, se pregunta cuáles serían los operadores de esos pliegues, que van a producir en un momento una mutación en la subjetividad. Allí Guattari relata la situación de una pequeña mutual de estudiantes, desde la cual algunos participantes produjeron pequeñas transformaciones que llevaron a otras, luego transformaciones mayores. Esos estudiantes, que disponían de fondos de la seguridad social de los estudiantes, crearon un gabinete de ayuda psicológica, inspirándose en el psicoanálisis, planteando problemas del saber, de la relación pedagógica, de la sexualidad de los estudiantes. "A partir de allí se cristalizaron lo que llamo operadores de subjetivación completamente nuevos" (Deleuze, 2015, p. 159). Estos operadores de subjetivación luego tuvieron su efecto en 1968: en Estrasburgo, un pequeño grupo desvió parte de esos fondos para imprimir octavillas, pequeños panfletos, que se repartieron en las jornadas previas a las revueltas del mayo francés. Dice Guattari "Hizo falta que hubiese un operador mínimo, un pequeño territorio de manejo de las relaciones actuales, podemos llamarlo un pequeño territorio liberado, un pequeño territorio de reapropiación, para que de repente el pliegue cuaje" (Deleuze, 2015, p.160).

\section{Discusión: Los grupos de estudio como pliegues}

Consideramos que los grupos de discusión, las pequeñas organizaciones de profesionales en plena dictadura, preservaron ese pequeño territorio de subjetividad, lo sustrajeron de los ámbitos donde el poder se hacía sentir con fuerza, de las universidades intervenidas, de los libros quemados y prohibidos, de los autores silenciados. El pensamiento que se quería desterrar definitivamente pudo ser resguardado, para quienes se quedaron en el país, atravesando los difíciles años de la dictadura militar. Con la recuperación de la democracia, volvieron a aparecer las ideas, así como los sujetos que debieron callarse y resguardarse en un exilio interno y los que volvieron del exilio propiamente dicho.

Las referencias a Deleuze y a Foucault, nos ayudan a analizar el movimiento de estas agrupaciones, de estos grupos que se reunían a leer, buscando un lugar y una forma para pensar juntos, para no quedarse solos y continuar transitando un camino. Estas referencias son tanto más necesarias en tanto y en cuanto la consideración de nuestra historia reciente como campo de estudio nos presenta un doble desafío: historizar el pasado acontecido y simultáneamente, reconstruir las condiciones de posibilidad de su propio análisis (Franco y Levín, 2007; Levin, 2016). 
Considerar las acciones ligadas a los mecanismos de discusión en el campo psi presentes en aquel momento, como una forma de pliegue en esa opresiva línea de poder, como un espacio donde algo de lo vital se hizo posible, que permitió que esas lecturas siguieran con vida, permite destacar un pequeño espacio donde el hacer y pensar con otros fuera mantenido. Pliegues, pequeño territorio liberado, para aquellos a quienes el poder pretendía imponer, erradicar todo vestigio de pensamiento propio. En este trabajo, recuperando voces de los actores que participaron de estos grupos, en Buenos Aires, Rosario y también en nuestro medio, pretendimos reflexionar sobre estas iniciativas que permitieron sostener la vitalidad de ciertas lecturas. Una resistencia ejercida, no como una confrontación en la línea del saber-poder, sino en puntos singulares, puntos de subjetivación de pequeñas experiencias instaladas en pliegues que se sustraen al saber-poder imperante. Estos espacios permitían dialogar y discutir con otros, romper el silencio que pesaba sobre el colectivo profesional; el foco no estará en grandes experiencias revolucionarias, sino en pequeñas experiencias locales, casi silenciosas, que lograron dar continuidad a algunas prácticas, ponerlas en común y ejercer una capacidad crítica sobre las mismas en relación a ciertas lecturas, que en definitiva resulta un cuestionarse acerca del orden de las cosas.

Como indicamos más arriba, Guattari relata dos experiencias muy acotadas que tuvieron un papel importante en el mayo del '68 en Francia, la de Estrasburgo y otro grupo en Nanterre: "Lo que quiero decir al subrayar estos dos ejemplos aparentemente un poco incomprensibles, es que justamente porque eran incomprensibles, justamente porque no eran directamente interpretables en las coordenadas políticas de la época, en las coordenadas sindicales, en las coordenadas grupusculares, crearon ese efecto de ruptura..." (Deleuze, 2015, p.160). Retomando las experiencias locales, justamente por ser silenciosas, por ser de baja magnitud, se tornaron incomprensibles para políticas imperantes y permitieron habitar de otro modo el espacio casi totalmente ocupado por el saber-poder impuesto por la dictadura.

Para finalizar, nos gustaría destacar estas palabras de Diana Maffía:

"Le debo tanto a Sadaf, le debo tanto a la convicción durante el exilio interno de quienes fueron echados o renunciaron a sus cátedras en la noche de los bastones largos, pero no renunciaron a su vocación y hasta el retorno de la democracia en los 80 mantuvieron generosamente vivo el espíritu crítico, la pasión por el conocimiento, la convicción ética como marco de la investigación, el compromiso con la democracia y los derechos humanos. Ellos 
nos convencieron, a quienes éramos tan jóvenes entonces, de mantener la persistencia en las ideas”. (Maffia, 2010, p.11)

\section{Referencias Bibliográficas}

Braunstein, N., Pasternac, M., Saal, F. y Benedito, G. (1976). Psicología: ideología y ciencia. México: Siglo XXI.

Calabresi, C. (2015). Los orígenes de la psicología en Mendoza.(Tesis doctoral inédita). Universidad Nacional de San Luis, San Luis.

Di Doménico, C., Giuliani, F., Visca, J., Ostrovsky, A., Moya, L.y Manso, L. (2008). A veinte años de la apertura de la carrera de Psicología en la Universidad Nacional de Mar del Plata: algunas reflexiones. Perspectivas en Psicología, 5, 24-32.

Casola, N. (octubre, 2009). A la deriva. El exilio interno bajo el terrorismo de Estado en Argentina. Actas del II Seminario Internacional Políticas de la Memoria:Vivir en dictadura. La vida de los argentinos entre 1976 y 1983. Buenos Aires, CCHCONTIArchivo Nacional de la Memoria -Secretaría de Derechos Humanos de la Nación.

Carpintero, E., y Vainer, A. (2005). Las huellas de la memoria: psicoanálisis y salud mental en la Argentina de los' 60 y'70.(Tomo II). Buenos Aires: Topía.

Catela Da Silva, L. (2001). No habrá flores en la tumba del pasado: la experiencia de reconstrucción del mundo de los familiares de desaparecidos. La Plata: Al Margen Ediciones.

Courel, R., y Talak, A. M. (2001). La formación académica y profesional del psicólogo en Argentina. En J. P. Toro y J. F.Villegas (Eds.) Problemas centrales para la formación académica y el entrenamiento profesional del psicólogo en las Américas. (Vol. 1, pp. 1-15). Buenos Aires: SIP.

Deleuze, G. (2015). La subjetivación, curso sobre Foucault. (Tomo III). Buenos Aires: Cactus.

Franco, M. y Levin, F. (2007) Historia reciente. Perspectivas y desafíos para un campo en construcción. Buenos Aires: Paidós. 
Foucault, M. (1988). El sujeto y el poder. Revista Mexicana de Sociología, 50(3), 3-20.

Recuperado de

http://www.peu.buap.mx/web/seminario_cultura/El_sujeto_y_el_poder.pdf

Gentile, A. (2009). El Centro de Estudios Psicoanalíticos (CEP) de Rosario, 1972-1975. Aporte a la reconstrucción de su historia. Revista Temas de Historia de la Psiquiatría Argentina, 28, 5-10.

González Aguirre, A. (2012). Tejiendo tramas de la memoria: aportes a la construcción de la identidad del colectivo de psicólogos de Córdoba. Córdoba: CCPP.

Jensen, S. (noviembre 2018). El Estado terrorista argentino y sus políticas sobre "prófugos" y “opcionados”. En M. S. Lastra yS. I. Jensen (Coords.) IV Jornadas de Trabajo sobre Exilios Políticos del Cono Sur en el siglo XX. Agendas, problemas y perspectivas conceptuales. Bahía Blanca: Departamento de Humanidades, Universidad Nacional del Sur.

Klappenbach, H. A. (2000). El título profesional de psicólogo en Argentina. Antecedentes históricos y situación actual. Revista Latinoamericana de Psicología, 32(3), 419446. Recuperado de https://www.redalyc.org/pdf/805/80532301.pdf

Klappenbach, H. A. (2006). Periodización de la psicología en Argentina. Revista de Historia de la Psicología, 27(1), 109-164. Recuperado de http://www.cepsifotocopiadora.com.ar/archivos/folios/37757_2015828.pdf

Klappenbach, H. A. (2015). La formación universitaria en psicología en Argentina: perspectivas actuales y desafíos a la luz de la historia. Universitas Psychologica, 14(3).http://dx.doi.org/10.11144/Javeriana.upsy14-3.fupa

Jensen, S. y Lastra, S (2014). La criminalización judicial de la militancia y su impacto en el retorno de los exiliados argentinos en la posdictadura. En M.S. Jensen y S.I. Lastra (Eds).Exilios: Militancia y represión. Nuevas fuentes y nuevos abordajes de los destierros de la Argentina de los años setenta(pp. 309-344). La Plata: EDULP. Recuperado de http://www.memoria.fahce.unlp.edu.ar/libros/pm.371/pm.371.pdf

Levín, F. (2016). El problema del sentido en la historia argentina reciente. Apuntes teóricometodológicos para el estudio de los procesos sociales de subjetivación de la experiencia del terrorismo de Estado. Papeles de Trabajo. La revista electrónica 
Exilio interno y campo psi en Córdoba durante la última dictadura militar.

Primeras aproximaciones e interrogantes

del IDAES,10 (17), 148-160. Recuperado de $\underline{\text { https://dialnet.unirioja.es/servlet/articulo?codigo }=5875344}$

Maffia, D. (2010). El análisis filosófico y la universidad de las catacumbas. Actas XV Congreso Nacional de Filosofía. Buenos Aires: AFRA.

Ferrero, L Quiroga, R.E. y Zuñiga, S. (2018). Ideas del Movimiento Social Crítico y su influencia en las prácticas de psicólogos en comunidades de la ciudad de Córdoba, en la década de 1970. Actas XIX Encuentro Argentino de Historia de la psiquiatría, la psicología y el psicoanálisis. Córdoba: Universidad Nacional de Córdoba.

Philp, M. (2009). Memoria y política en la historia argentina reciente: una lectura desde Córdoba.Córdoba: Universidad Nacional de Córdoba.

San Nicolás, N. (2016). Aniquilar la oposición. El terrorismo de Estado en la UNC: contexto y expresiones. En S. Romano (Ed.). Colectivos y parcialidades políticas y sociales: los desaparecidos y asesinados de Córdoba en los '70 (pp.27-60).Córdoba: Editorial Facultad de Filosofía y Humanidades, Universidad Nacional de Córdoba.

Sanz Ferramola, R. (2000). La psicología como ideología exótica en los oscuros años del proceso de desorganización nacional: 1975-1980. Fundamentos en Humanidades, (2), 43-62.

Schiavoni, L. y Wouters, R. (2014). La comunidad terapéutica: Más allá de una experiencia piloto, el clima de época y la resistencia democratizadora. (Tesina de grado inédita). Universidad Nacional de Córdoba, Córdoba

Servetto, A. M. (2004). Córdoba en los prolegómenos de la dictadura La política del miedo en el gobierno de Lacabanne. Estudios: Revista del Centro de Estudios Avanzados, $(15)$, 143-156. Recuperado de https://revistas.unc.edu.ar/index.php/restudios/article/view/13542/0 